\title{
Nonmanipulable voting schemes when participants' interests are partially decomposable*
}

\author{
Diego Moreno and Mark Walker \\ Department of Economics, University of Arizona, Tucson, AZ 85721, USA
}

Received October 2, 1989 / Accepted February 4, 1991

\begin{abstract}
Recent papers by Barberá and Peleg and by Zhou have established that the Gibbard-Satterthwaite Theorem remains valid when individuals are restricted to reporting only "reasonable" preferences. We present a theorem that covers situations in which, as in Barberá-and-Peleg and Zhou, preferences may be restricted to reasonable ones, but in which, additionally, it may be known in advance that some dimensions of the social decision do not affect all the participants - i.e., in which the social decisions are partially decomposable into decisions that affect only subsets of the participants. As in the previous theorems, the conclusion of this new theorem is that nonmanipulable voting schemes must be dictatorial.
\end{abstract}

The Gibbard-Satterthwaite Theorem tells us that a voting scheme must be either manipulable or dictatorial if it admits all possible preferences as individual strategies. Recent papers by Barberá and Peleg (1990) and by Zhou (1991) have established that the Gibbard-Satterthwaite Theorem remains valid even when individuals are restricted to reporting only "reasonable" preferences. There remains, however, a broad class of collective decision situations that are not covered by the original theorem or by the two newer ones - namely, situations in which some dimensions of the alternative social decisions are known to affect only a subset of the participants in the decision process. We present a theorem that covers a class of such situations: We will show that the fundamental impossibility conclusion of the previous theorems remains valid when the alternative social decisions can be expressed in terms of some dimensions that are known to affect all participants (as in the previous theorems) and other dimensions that are known to affect only single participants or subsets of participants.

The Gibbard-Satterthwaite Theorem, as originally stated and proved by Gibbard (1973) and Satterthwaite (1975), states that if a voting scheme allows each

\footnotetext{
* An early version of this paper was delevered at the 1989 NBER-NSF Conference on Decentralization at $\mathrm{Cal}$ Tech; comments by the conference participants are appreciated, as are comments by James Schmitz.
} 
of its participants to claim that his preference is any preorder over the set of alternatives, and if the set of alternatives is finite, then any voting scheme whose range contains more than two distinct outcomes must be either manipulable or dictatorial. The theorem in its original form thus left open the possibility that if the set of alternatives has some natural structure (for example, an order, linear, and /or topological structure), then it might be possible to design a voting scheme that performs more satisfactorily if we require that participants' behavior be rationalizable by "reasonable" or "plausible" preferences - by preferences, in other words, that are consistent with the structure of the set of alternatives. The Barberá-and-Peleg (henceforth B \& P) and Zhou papers establish that even if we allow only continuous preferences (Barberá and Peleg) or only strictly convex preferences (Zhou), every voting scheme will still be either manipulable or dictatorial.

We shall consider the following problem, which leads to a further natural restriction on the set of admissible individual preferences. Suppose that one knows, when designing a voting mechanism, that some of the participants will not "care about" some dimensions of the decision, (i.e., their utility functions will not be affected by some components). The classical instance of this in economics is of course the problem of allocating goods to individuals when any one individual's consumption has no effect upon any other individual's utility. More generally, the idea that there are certain identifiable, potentially agreed-upon features of the world that affect some individuals and not others, and that only those individuals who are affected should be able to influence the choice of those features, is the cornerstone of Western liberal thought. If we know that some of the participants in the social decision will not be affected by certain dimensions of the decision, then to what extent, if any, can we exploit this knowledge to design a voting scheme that is neither manipulable nor dictatorial?

It is intuitively clear, for example, that if each participant cares about different dimensions of the decision, then perfectly good outcomes will be achieved by "decentralizing" the decision-making: we simply allow each participant to choose the values of the components that he, and only he, cares about. Conversely, when every participant cares about every dimension, the B \& $\mathbf{P}$ and Zhou theorems tell us that any nonmanipulable voting scheme will be dictatorial. In other words, if the participants' interests can never be in conflict, then a satisfactory ${ }^{1}$ voting scheme exists, and if, at the other extreme, there is potential for the participants' interests to be "fully" in conflict, then no satisfactory scheme exists. The question we will address is whether satisfactory voting schemes exist when it is known that the participants' interests are less than fully "decomposable". What degree of "overlap" in the dimensions that different individuals care about is too much to permit the existence of any satisfactory voting scheme? The theorem we will present provides a partial answer to this question. Roughly speaking, the theorem states that if there are some dimensions that affect only some of the individuals, and if the remaining dimensions affect everyone, then any voting scheme that satisfies a very weak unanimity condition, and whose range includes a two-

\footnotetext{
1 The term "satisfactory" is to be understood informally at this stage. The "completely decentralized" scheme applied to the "completely decomposable" environments is technically dictatorial - indeed, each participant always gets exactly what he wants - but is is clearly quite satisfactory; therefore, we do not want to simply define "satisfactory" voting schemes to be those that are neither manipulable nor dictatorial. See also Examples 4 and 5 in this connection.
} 
dimensional set of decisions that affect everyone, must be either manipulable or dictatorial.

The remainder of the paper is organized as follows. We will first lay out the notation and definitions we will need, followed by the theorems's two central assumptions. The assumptions characterize formally the kinds of social decision problems that are covered by the theorem, and we provide several illustrative examples of social decision problems that satisfy the assumptions. The theorem will then be stated and proved, and then we conclude with two additional examples and several remarks concerning the theorem's limitations and its potential generalizations.

\section{Definitions}

1. $Z$ denotes the set of (feasible) alternatives and $N$ denotes the set $\{1, \ldots, n\}$ of individuals.

2. A utility function is a real-valued function on $Z$. For each $i \in N$, a set $U_{i}$ of utility functions $u_{i}: Z \rightarrow \mathbb{R}$ is given. The members of $U_{i}$ are referred to as the admissible utility functions for $i$. $\mathscr{U}$ denotes the Cartesian product $\Pi_{i=1}^{n} U_{i}$; the members $\mathbf{u}=\left(u_{1}, \ldots, u_{n}\right)$ of $\mathscr{U}$ are called profiles. If $\mathbf{u}$ is a profile and $\tilde{u}_{i}$ is a member of $U_{i}$, then $\left(\tilde{u}_{i}, \mathbf{u}_{\sim i}\right)$ denotes the profile in which $\tilde{u}_{i}$ has replaced the $i^{\text {th }}$ component of $\mathbf{u}$. For each $i \in N, \mathscr{U}_{\sim i}$ denotes the Cartesian product $\Pi_{j \neq i} U_{j}$.

3. A voting scheme, or mechanism, is a function $f: \mathscr{U} \rightarrow Z$. The alternative $f(\mathbf{u})$ is called the outcome associated with $\mathbf{u}$. We will simplify notation slightly by writing $u_{i} f(\mathbf{v})$ for $u_{i}(f(v))$, i.e., for $i$ 's utility (according to the utility function $u_{i}$ ) at the outcome associated with profile $\mathbf{v}$.

4. A mechanism $f$ is manipulable by individual $i$ at profile $\mathbf{u}$ via utility function $\tilde{u}_{i}$ if $u_{i} f\left(\tilde{u}_{i}, \mathbf{u}_{\sim i}\right)>u_{i} f(\mathbf{u})$. A mechanism $f$ is nonmanipulable if, for each profile $\mathbf{u} \in \mathscr{U}$, each $i \in N$, and each $\tilde{u}_{i} \in U_{i}, f$ is not manipulable by $i$ at $\mathbf{u}$ via $\tilde{u}_{i}$.

5. An individual $i \in N$ is a dictator for the mechanism $f$ if for every profile $\mathbf{u}=\left(u_{1}, \ldots, u_{n}\right) \in \mathscr{U}$, the outcome associated with $\mathbf{u}$ maximizes $u_{i}$ on $f(\mathscr{U})$. A mechanism $f$ is dictatorial if there is a dictator for $f$, otherwise it is nondictatorial.

The theorems of Gibbard, Satterthwaite, Barberá and Peleg, and Zhou all give conditions under which every nonmanipulable mechanism is dictatorial: viz., if the range of $f$ contains at least three elements and each $U_{i}$ is the set of all utility functions on $Z$ (Gibbard and Satterthwaite); if $Z$ is a metric space, the range of $f$ contains at least three elements, and each $U_{i}$ contains all the continuous real-valued functions on $Z$ (Barberá-and-Peleg); and if $Z$ is a compact convex subset of a finite-dimensional Euclidean space, with $\operatorname{dim}($ Range $f) \geqq 2$, and each $U_{i}$ contains all the strictly concave quadratic functions on $Z$ ( $Z$ hou).

None of the above-mentioned theorems, however, applies to the following example. (Nor do they apply to Examples 2 and 3, to be presented shortly.)

Example 1. $Z=X \times Y$, where $X$ is the unit square in $\mathbb{R}^{2}$ and $Y$ is the set $\left\{y \in \mathbb{R}_{+}^{3} \mid y_{1}+y_{2}+y_{3} \leqq 1\right\}$. For each $i \in N=\{1,2,3\}$, let $\dot{U}_{i}$ denote the set of all strictly concave real-valued functions $\dot{u}_{i}$ on $X \times[0,1]$, and let $U_{i}$ be the set of all functions $u_{i}: Z \rightarrow \mathbb{R}$ of the form $u_{i}(x, y)=\dot{u}_{i}\left(x, y_{i}\right)$ for $\dot{u}_{i} \in \dot{U}_{i}$. 
In the example, each individual $i \in N$ is always (i.e., whatever his utility function $\dot{u}_{i}$ ) "unaffected" by a change in $y_{j}$ if $j \neq i$. But in each of the above-mentioned theorems each set $U_{i}$ is assumed to include a substantial class of utility functions that are affected by changing any of an alternative's components. Indeed, the theorems do not apply even if we change the example so that it is "decomposable" in the $Y$-components - i.e., if we let $Y$ be the unit cube in $\mathbb{R}^{3}$.

The theorem we are about to present covers the kind of situation represented by the example - situations in which there are some dimensions of the social choice that directly affect only some of the individuals. The theorem tells us that the fundamental incompatibility between nonmanipulability and nondictatorship is not alleviated by knowing in advance that some of the participants do not care about certain features of the social alternatives.

The following two assumptions characterize the kinds of social decision problems that the theorem will cover. Assumption 1 captures the idea that is is known when designing a voting scheme that the social alternatives have some features that do not affect everyone. Assumption 2 specifies that each set $U_{i}$ of admissible utility functions must at least include all utility functions that are quadratic and strictly concave in the components that affect individual $i$ 's utility.

Assumption 1. (1a) $Z=X \times Y$ and $Y \subseteq Y_{1} \times \cdots \times Y_{n}$, where $X$ and $Y_{1}, \ldots, Y_{n}$ are compact convex subsets of finite-dimensional Euclidean spaces, and where each $Y_{i}$ is the $i^{\text {th }}$ projection of $Y-i$.e., if $y_{i} \in Y_{i}$, then $y_{i}$ is the $i^{\text {th }}$ component of some $y \in Y$.

(1b) Every admissible $u_{i}$ depends only upon $x \in X$ and $y_{i} \in Y_{i}-$ i.e., for each $i \in N$ and each $u_{i} \in U_{i}$, there is a function $\dot{u}_{i}: X \times Y_{i} \rightarrow \mathbb{R}$ for which $u_{i}(x, \mathbf{y})=\dot{u}_{i}\left(x, y_{i}\right)$.

Assumption 1 seems to imply that the $y$-component of the social decision must be "decomposable" into components $y_{i}$ that only single individuals care about that is, the assumption seems to require that each component of the social decision be cared about either by all the participants or by only a single participant. The following example shows that because Assumption 1a allows the set $Y$ to be a proper subset of $Y_{1} \times \cdots \times Y_{n}$, the assumption is not nearly so restrictive.

Example 2. A club operates a swimming pool and a spa. Each member of the club uses the pool, and therefore each member cares about both the temperature of the pool's water, $x_{t}$, and its level of chlorination, $x_{c}$. Only a subset $S$ of the members uses the spa, however, and it is therefore known that only the members of $S$ care about the temperature of the water in the spa.

Let $X$ denote the set of feasible pool-temperature-and-chlorination pairs $\left(x_{t}, x_{c}\right)$ in the example; let $Y^{*}$ denote the set of feasible temperatures for the spa; for each $i \in S$, let $Y_{i}=Y^{*}$; and for each $i \notin S$, let $Y_{j}$ be any singleton. Let $Y=\left\{y \in Y_{1} \times \cdots \times Y_{n} \mid i, j \in S \Rightarrow y_{i}=y_{j}\right\}$. Then $X \times Y$ is the set of feasible decisions (Assumption 1a is satisfied), and the admissible utility functions $u_{i}$ will satisfy Assumption 1b. (The theorem will cover situations in which, as in the example, the dimension of $X$ is at least two.)

Some notation. Henceforth, Assumption 1 will always be satisfied, and we will therefore use the notation $\dot{u}_{i}$ defined in Assumption $1 \mathrm{~b}$, as well as the following: $f_{x}(\mathbf{u})$ will denote the $x$-component of $f(\mathbf{u}) ; f_{y}(\mathbf{u})$ will denote the $y$-component of $f(\mathbf{u})$; and for each $i \in N, f_{i}(\mathbf{u})$ will denote the $y_{i}$-component of $f(\mathbf{u})$. For 
each $i \in N, U_{i}^{Q}$ will denote the set of all functions on $X \times Y$ that satisfy Assumption $1 \mathrm{~b}$ and for which $\dot{u}_{i}$ is strictly concave and quadratic. The set of all strictly concave quadratic real-valued functions on $X$ will be denoted by $V$, and for every $\mathbf{v} \in V$, every $i \in N$, and every $a_{i} \in Y_{i}, v^{a_{i}}$ will denote the utility function on $X \times Y$ defined as follows:

$$
v^{a_{i}}(x, y)=v(x)-\left\|y_{i}-a_{i}\right\|^{2} .
$$

For each $i \in N, U_{i}^{*}$ will denote the set of all such utility functions (thus, $U_{i}^{*}$ can be written $V \times Y_{i}$ ), and $\mathscr{U}^{*}$ will denote the set of all profiles of such functions for which a $\in Y: \mathscr{U}^{*}=V^{n} \times Y$. Note that $U_{i}^{*} \subseteq U_{i}^{Q} \subseteq U_{i}$ for each $i \in N$, and that if $Y$ is a proper subset of $\Pi_{1}^{n} Y_{i}$, then $\mathscr{U}^{*}$ is a proper subset of $\Pi_{1}^{n} U_{i}^{*}$.

Assumption 2. For each $i \in N, U_{i}^{Q} \subseteq U_{i}$; that is, each set $U_{i}$ includes all utility functions $u_{i}$ that satisfy Assumption $1 \mathrm{~b}$ and for which $\dot{u}_{i}$ is strictly concave and quadratic.

Assumption 2 requires, in particular, that the $U_{i}$ include non-separable utility functions. Example 4 shows that this requirement is essential.

The following example describes a concrete decision situation which satisfies Assumptions 1 and 2, and which therefore falls within the scope of the theorem we are about to present (and it is not covered by previous theorems).

Example 3. A community of $n$ farmers controls two water reservoirs, which we label $A$ and $B$. Everyone uses Reservoir $A$ for recreational pursuits (fishing, boating, swimming, etc.), and the water in Reservoir $B$ is used to irrigate all the farmers' crops. Everyone therefore cares about both the water level and the degree of chlorination in Reservoir $A$, and each farmer cares only about his own allocation of water from Reservoir $B$.

In this example, the water level $x_{w}$ and the degree of chlorination $x_{c}$ in Reservoir $A$ are pure public goods: Each farmer would be assumed to have a preference over alternative pairs $\left(x_{w}, x_{c}\right)$, and although we might expect such preferences to be single-peaked or quasi-concave, we would not expect them to necessarily be monotone. The allocation $\left(y_{1}, y_{2}, \ldots, y_{n}\right)$ of some or all of the water in Reservoir $B$ must satisfy $y_{1}+y_{2}+\ldots+y_{n} \leqq y$; it is natural to assume that each farmer $i \in\{1,2, \ldots, n\}$ has a preference (single-peaked, but not necessarily monotone) that depends only on $y_{i}$ and not on $y_{j}$ for $j \neq i$. The set of feasible decisions is $X \times Y$, where $X$ is a subset of the product of two real intervals and $Y=\left\{\left(y_{1}, \ldots, y_{n}\right) \in \mathbb{R}_{+}^{n} \mid \Sigma y_{i} \leqq y\right\}$.

The example clearly satisfies Assumption 1, and Assumption 2 is quite reasonable. On the other hand, some natural variations of the example violate the assumptions. Assumption 1a, for example, requires that the set of feasible decisions be the Cartesian product $X \times Y$. Thus, if the water levels in $A$ and $B$ cannot be set independently of one another, Assumption 1a will be violated. Assumption $1 \mathrm{~b}$ would be violated if the farmers could obtain "recreational" use (in addition to irrigation) from the water in $B$. Assumption 2 does not allow us to restrict our attention to monotone preferences over any of the components of the decision; in particular, then, the quantities $y_{i}$ cannot represent monetary transfers (instead of irrigation levels) or any other commodity of which the farmers are known to always prefer more to less. See also Remarks 2 and 4 at the paper's conclusion concerning these limitations. 
When the social choice problem has the kind of separable structure described by Assumption 1, then the unanimity feature of nonmanipulable mechanisms a feature that is fundamental in the proofs given by Barberá and Peleg and by Zhou - no longer holds. The proof we are about to present makes use of a more limited unanimity property, one that we will refer to as "conditional unanimity." This property is akin to the "nonwastefulness" property generally considered desirable in allocation mechanisms, and is clearly substantially weaker than the standard notion of unanimity (viz., that when all utility functions are maximized at the same $z \in Z$, then that $z$ is the mechanism's outcome). In particular, when $Y$ is a singleton - as in B \& $\mathrm{P}$ and Zhou, for example - conditional unanimity is vacuous.

The CU property. A mechanism $f$ has the Conditional Unanimity (CU) Property if, whenever $\mathbf{a} \in Y$ and, for each $i \in N$, the component $a_{i}$ of $\mathbf{a}$ is the unique maximizer of $\dot{u}_{i}\left(f_{x}(\mathbf{u}), \cdot\right)$, then $f_{y}(\mathbf{u})=\mathbf{a}$.

There is one additional concept that we will find helpful in developing a proof of the theorem:

$X$-Dictatorship. We say that individual $k$ is an $X$-dictator for $f$ (and we say that $f$ is $X$-dictatorial $)$ if $u_{k} f(\mathbf{u}) \geqq u_{k}\left(x, f_{y}(\mathbf{u})\right)$ for every $u \in \mathscr{U}$ and every $x \in f_{x}(\mathscr{U})$.

Theorem. If the set $Z$ of social alternatives and the sets $U_{1}, \ldots, U_{n}$ of utility functions satisfy Assumptions 1 and 2 , and if $n \geqq 2$, then every nonmanipulable mechanism $f$ that has the CU property, and for which the set $f_{x}(\mathscr{U})$ has dimension at least 2 , is dictatorial.

The proof of the theorem will proceed via several lemmas. Throughout the proof we will maintain the assumptions of the theorem, and we assume that $f: \mathscr{U} \rightarrow Z$ is a nonmanipulable mechanism that has the CU property. For each $\mathbf{a} \in \Pi_{1}^{n} Y_{i}$, define the mechanism $f^{\mathbf{a}}: V^{n} \rightarrow X$ as follows: $\forall \mathbf{v} \in V^{n}: f^{\mathbf{a}}(\mathbf{v})=f_{x}\left(\mathbf{v}^{\mathbf{a}}\right)$. Note, too, that although the restriction of a voting scheme $f$ to a subset $U$ of $\mathscr{U}$ is not ifself generally a voting scheme, we will nevertheless say that $f$ is nonmanipulable or dictatorial or $X$-dictatorial on $U$ if the defining condition is satisfied on $U$.

Lemma 1 is an immediate consequence of the CU property and is used repeatedly throughout the proof. Lemma 2 establishes that each $f^{\mathbf{a}}$ is nonmanipulable. Lemma 3 establishes that each $f^{\mathbf{a}}$ attains the same set of $x$-outcomes as $f$-i.e., $f^{\mathbf{a}}\left(V^{n}\right)=f_{x}(\mathscr{U})$ for each $\mathbf{a} \in Y$. Lemma 4 establishes that each $f^{\mathbf{a}}$ is dictatorial. Lemma 5 establishes that all the outcomes attainable via $f$ on the domain $\mathscr{U}$ can be attained on the restricted domain $\mathscr{U}^{*}$. Lemma 6 establishes that $f$ is $X$-dictarorial on $\mathscr{U}^{*}$. We then expand the domain of profiles from $\mathscr{U}^{*}$ "almost" to $\mathscr{U}$ and show in Lemma 7 that some individual is an $X$-dictator on this larger domain. Finally, in Lemma 8 we show that some individual is actually a dictator (not merely an $X$-dictator) on a certain subdomain of $\mathscr{U}$, and it is then easy to complete the theorem's proof by showing that the same individual is in fact a dictator on $\mathscr{U}$.

Lemma 1. For each $i \in N$, each $\mathbf{v} \in V^{n}$, and each $\mathbf{a} \in Y, f_{i}\left(\mathbf{v}^{\mathbf{a}}\right)=a_{i}$ and $v_{i}^{a_{i}} f\left(\mathbf{v}^{\mathbf{a}}\right)=v_{i} f^{\mathbf{a}}(\mathbf{v})$.

Lemma 2. For each $\mathbf{a} \in Y, f^{\mathbf{a}}$ is nonmanipulable. 
Proof. Suppose $f^{\mathbf{a}}$ were manipulable for some $\mathbf{a} \in Y$; i.e., for some $i \in N$, some $\checkmark \in V^{n}$, and some $\tilde{v}_{i} \in V$, the following inequality holds:

$$
v_{i} f^{\mathbf{a}}\left(\tilde{v}_{i}, v_{\sim i}\right)>v_{i} f^{\mathbf{a}}(\mathbf{v}) \text {. }
$$

But then, according to Lemma 1, we have

$$
\begin{aligned}
v_{i}^{a_{i}} f\left(\tilde{v}_{i}^{a_{i}}, \mathbf{v}_{\sim i}^{\mathbf{a}}\right) & =v_{i} f_{a}\left(\tilde{v}_{i}^{a_{i}}, \mathbf{v}_{\sim i}^{\mathbf{a}}\right)-\left\|a_{i}-a_{i}\right\|^{2} \\
& =v_{i} f^{\mathbf{a}}\left(\tilde{v}_{i}, \mathbf{v}_{\sim i}\right)>v_{i} f^{\mathbf{a}}(\mathbf{v})=v_{i}^{a_{i}} f\left(\mathbf{v}^{\mathbf{a}}\right),
\end{aligned}
$$

and therefore individual $i$ can manipulate $f$ at $\mathbf{v}^{\mathbf{a}}$ via $\tilde{v}_{i}^{a_{i}}$, contrary to the assumption that $f$ is nonmanipulable.

Lemma 3. For each $\mathbf{a} \in Y, f^{\mathbf{a}}\left(V^{n}\right)=f_{x}(\mathscr{U})$.

Proof. Clearly, $\left.f^{\mathbf{a}}\left(V^{n}\right) \subseteq f_{x} \mathscr{U}\right)$. In order to show that $f_{x}(\mathscr{U}) \subseteq f^{\mathbf{a}}\left(V^{n}\right)$, suppose to the contrary that there exist an $\mathbf{a} \in Y$ and $\mathbf{a} \overline{\mathbf{u}} \in \mathscr{U}$ for which $f(\overline{\mathbf{u}}) \notin f^{\mathbf{a}}\left(V^{n}\right)$. Let $x^{0}=f_{x}(\overline{\mathbf{u}})$. It is easy to show that because $f^{\mathbf{a}}$ is nonmanipulable $f^{\mathbf{a}}\left(V^{n}\right)$ is a closed set (see the proof of "Step 2" in Zhou); consequently, there is $\delta_{0}>0$ such that

$$
\left\|x-x^{0}\right\|<\delta_{0} \Rightarrow x \notin f^{\mathbf{a}}\left(V^{n}\right) .
$$

Let $v_{1}$ be a member of $V$ that satisfies both

$$
\begin{aligned}
& x^{0} \text { maximizes } v_{1}, \quad \text { and } \\
& \left\|x-x^{0}\right\| \geqq \delta_{0} \Rightarrow v_{1}\left(x^{0}\right)-v_{1}(x)>\max _{y_{1} \in Y_{1}}\left\|y_{1}-a_{1}\right\|^{2},
\end{aligned}
$$

and let $\left(x^{1}, y_{1}^{1}\right)$ denote $\left(f_{x}\left(v_{1}^{a_{1}}, \bar{u}_{\sim 1}\right), f_{1}\left(v_{1}^{a_{1}}, \bar{u}_{\sim 1}\right)\right)$. Then we have

$$
\begin{aligned}
v_{1}\left(x^{1}\right) & \geqq v_{1}\left(x^{1}\right)-\left\|y_{1}^{1}-a_{1}\right\|^{2} \\
& =v_{1}^{a_{1}} f\left(v_{1}^{a_{1}}, \bar{u}_{\sim 1}\right) \\
& \geqq v_{1}^{a_{1}} f(\overline{\mathbf{u}}), \quad \text { because } f \text { is nonmanipulable }, \\
& =v_{1}\left(x^{0}\right)-\left\|f_{1}(\overline{\mathbf{u}})-a_{1}\right\|^{2} \\
& \geqq v_{1}\left(x^{0}\right)-\max _{y_{1} \in Y_{1}}\left\|y_{1}-a_{1}\right\|^{2} .
\end{aligned}
$$

According to (3), then, we must have $\left\|x^{1}-x^{0}\right\|<\delta_{0}$, and therefore, according to $(1), x^{1} \notin f^{\mathbf{a}}\left(V^{n}\right)$.

Because $x^{1} \notin f^{\mathbf{a}}\left(V^{n}\right)$, there is a $\delta_{1}$ with which we can repeat the argument in the preceding paragraph, with $x^{1}$ and $\delta_{1}$ replacing $x^{0}$ and $\delta_{0}$, thereby obtaining a $v_{2} \in V$ and an $x^{2}=f_{x}\left(v_{1}^{a_{1}}, v_{2}^{a_{2}}, \bar{u}_{3}, \ldots, \bar{u}_{n}\right) \notin f^{\mathbf{a}}\left(V^{n}\right)$. Repeating the argument another $n-2$ times yields $x^{n}=f_{x}\left(\mathbf{v}^{\mathbf{a}}\right) \notin f^{\mathbf{a}}\left(V^{n}\right)$, a contradiction, which completes the proof of Lemma 3.

Lemma 4. For each $\mathbf{a} \in Y, f^{\mathbf{a}}$ is dictatorial.

Proof. Because each $f^{\mathbf{a}}$ is nonmanipulable, we need only establish that the dimension of the range of $f^{a}$ is at least two in order to apply Zhou's theorem, 
which yields the desired conclusion. But Lemma 3 established that the range of each $f^{\mathbf{a}}$ is $f_{x}(\mathscr{U})$, and it was assumed in the statement of the theorem that $f_{x}(\mathscr{U})$ has dimension at least two.

Lemma 5. $f\left(\mathscr{U}^{*}\right)=f(\mathscr{U})$.

Proof. Clearly, $f(\mathscr{U}) \subseteq f(\mathscr{U})$. To establish that $f(\mathscr{U}) \subseteq f\left(\mathscr{U}^{*}\right)$, let $(x, \mathbf{y})$ $\in f(\mathscr{U})$. Then clearly $x \in f_{x}(\mathscr{U})$ and $\mathbf{y} \in Y$. Because $x \in f_{a}(\mathscr{U})$, Lemma 3 ensures that there is a $\mathbf{v} \in V^{n}$ for which $f^{\mathbf{y}}(\mathbf{v})=x-$ i.e., $f_{x}\left(\mathbf{v}^{\mathbf{y}}\right)=x$. Lemma 1 yields $f_{y}\left(\mathbf{v}^{\mathbf{y}}\right)=\mathbf{y}$. We have $f\left(\mathbf{v}^{\mathbf{y}}\right)=(x, \mathbf{y})$, and since $\mathbf{v}^{\mathbf{y}} \in \mathscr{U}^{*}$, this completes the proof of Lemma 5 .

Lemma 6. $f$ is $X$-dictatorial on $\mathscr{U}^{*}$.

Proof. Let $\overline{\mathbf{a}} \in Y$ and, without loss of generality, let individual 1 be a dictator for $f^{\overline{\mathbf{a}}}$ (Lemma 4). We will show that individual 1 is an $X$-dictator for $f$ on $\mathscr{U}^{*}$. As a first step in the lemma's proof, we will show that at any $\mathbf{u}=\mathbf{v}^{\mathrm{a}} \in \Pi_{1}^{n} U_{i}^{*}$ for which $a_{1}=\tilde{a}_{1}$ and $v_{1}$ has a unique maximizer on the set $f_{x}\left(\mathscr{U}^{*}\right)=f_{x}(\mathscr{U})$, the $x$ outcome $f_{x}(\mathbf{u})$ will be that maximizer. Thus, let $\bar{v}_{1}$ be any member of $V$ that has a unique maximizer on $f_{x}(\mathscr{U})$, and denote the maximizer of $\bar{v}_{1}$ by $\bar{x}$. Of course, since individual 1 is a dictator for $f^{\overline{\mathbf{a}}}$, we have $f_{x}\left(\mathbf{v}^{\overline{\mathbf{a}}}\right)=\bar{x}$ for any $\mathbf{v} \in V^{n}$ such that $v_{1}=\bar{v}_{1}$. We must show that changing the components of $\overline{\mathbf{a}}$ (other than the first one) does not alter the $x$-outcome at any of these $\mathbf{v}$.

Let $\mathbf{a}=\left(\bar{a}_{1}, a_{2}, \ldots, a_{n}\right) \in \Pi_{1}^{n} Y_{i}$, and we will show that $f_{x}\left(\mathbf{v}^{\mathbf{a}}\right)=\bar{x}$ for any $\mathbf{v} \in V^{n}$ such that $v_{1}=\bar{v}_{1}$. The argument will be carried out recursively: First we change only the last component of $\mathbf{a}$ to the alternative value $a_{n}$; we write $\mathbf{a}^{\prime}$ for the $n$ tuple $\left(\bar{a}_{1}, \ldots, \bar{a}_{n-1}, a_{n}\right)$; and we show that $f_{x}\left(\mathbf{v}^{\mathbf{a}^{\prime}}\right)=\bar{x}$ for every $\mathbf{v} \in\left\{\bar{v}_{1}\right\} \times V^{n-1}$. Suppose to the contrary that $f_{x}\left(\mathbf{v}^{\mathbf{a}^{\prime}}\right)=\hat{x} \neq \bar{x}$ for some $\mathbf{v} \in\left\{\bar{v}_{1}\right\} \times V^{n-1}$. Then let $\hat{v}_{n}$ be a member of $V$ for which $\hat{v}_{n}(\bar{x})<\hat{v}_{n}(\hat{x})-\left\|\bar{a}_{n}-y_{n}\right\|^{2}$ for every $y_{n} \in Y_{n}$, and denote $\left(v_{1}, \ldots, v_{n-1}, \hat{v}_{n}\right)$ by $\hat{\mathbf{v}}$. Then $f_{x}\left(\hat{\mathbf{v}}^{\mathbf{a}}\right)=\bar{x}$, and we therefore have

$$
\begin{aligned}
\hat{v}_{n}^{\bar{a}_{n}} f\left(\hat{\mathbf{v}}^{\overline{\mathbf{a}}}\right) & =\hat{v}_{n}(\bar{x})-\left\|\bar{a}_{n}-\bar{a}_{n}\right\|^{2} \\
& =\hat{v}_{n}(\bar{x}) \\
& <\hat{v}_{n}(\hat{x})-\left\|\bar{a}_{n}-f_{n}\left(\mathbf{v}^{\mathbf{a}^{\prime}}\right)\right\| \\
& =\hat{v}_{n}^{\bar{a}_{n}} f\left(\mathbf{v}^{\mathbf{a}^{\prime}}\right) ;
\end{aligned}
$$

hence, individual $n$ can manipulate $f$ at $\hat{\mathbf{v}}^{\overline{\mathbf{a}}}$ via $v_{n}^{\alpha^{\prime} n}$, contradicting the assumption that $f$ is nonmanipulable. This establishes that $f_{x}\left(\mathbf{v}^{\mathbf{a}^{\prime}}\right)=\bar{x}$ after all - i.e., changing the last component of $\overline{\mathbf{a}} \mathrm{did}$ not change the $x$-outcome from $\bar{x}$ for any $\mathbf{v} \in\left\{\bar{v}_{1}\right\} \times V^{n-1}$. Now we can repeat the same argument for $k=n-1, n-2, \ldots, 2$, each time writing $\mathbf{a}^{\prime}=\left(\bar{a}_{1}, \ldots, \bar{a}_{k-1}, a_{k}, \ldots, a_{n}\right)$ and showing that $f_{x}\left(\mathbf{v}^{\mathbf{a}^{\prime}}\right)=\bar{x}$ for every $\mathbf{v} \in\left\{\overline{\boldsymbol{v}}_{1}\right\} \times V^{n-1}$. After the last step, $k=2$, we will have $\mathbf{a}^{\prime}=\left(\bar{a}_{1}, a_{2}, \ldots, a_{n}\right)=\mathbf{a}$ and $f_{x}\left(\mathbf{v}^{\mathbf{a}}\right)=\bar{x}$, as desired.

In order to complete the proof that individual 1 is an $X$-dictator on $\mathscr{U}^{*}$, we suppose to the contrary that he is not - i.e., that while we continue to assume that individual 1 is a dictator for $f^{\bar{a}}$, there is a member $\mathbf{v}^{\overline{\bar{a}}}$ of $\mathscr{U}^{*}$ for which $f_{x}\left(\mathbf{v}^{\overline{\mathbf{a}}}\right)$ does not maximize $v_{1}$ on $f_{x}\left(\mathscr{U}^{*}\right)=f_{x}(\mathscr{U})$. Then certainly individual 1 is not a dictator for $f^{\overline{\bar{a}}}$, and since $\overline{\overline{\mathbf{a}}} \in Y$, Lemma 4 allows us to assume, without 
loss of generality, that individual 2 is a dictator for $f^{\overline{\bar{a}}}$. Now let $\mathbf{a}=$ $\left(\bar{a}_{1}, \overline{\bar{a}}_{2},{ }_{2}, \ldots, \overline{\bar{a}}_{n}\right)$, and let $\mathbf{v}$ be a member of $V^{n}$ that satisfies each of the following:

$v_{1}$ has a unique maximizer $\bar{x}$ on $f_{x}(\mathscr{U})$;

$v_{2}$ has a unique maximizer $\overline{\bar{x}}$ on $f_{x}(\mathscr{U})$;

$\bar{x} \neq \overline{\bar{x}}$;

$v_{1}(\bar{x})-\left\|\overline{\bar{a}}_{1}-y_{1}\right\|^{2}>v_{1}(\overline{\bar{x}}), \quad$ for every $y_{1} \in Y_{1}$.

Note that $f_{x}\left(\mathbf{v}^{\mathbf{a}}\right)=\bar{x}$ (a consequence of (1) and the first step in the lemma's proof) and $f_{x}\left(\mathbf{v}^{\overline{\mathbf{a}}}\right)=\overline{\bar{x}}$ (a consequence of (2)). Therefore we have

$$
\begin{aligned}
v_{1}^{\overline{\bar{a}}_{1}} f\left(\mathbf{v}^{\overline{\bar{a}}}\right) & =v_{1}(\overline{\bar{x}})-\left\|\overline{\bar{a}}_{1}-\overline{\bar{a}}_{1}\right\|^{2} \\
& =v_{1}(\overline{\bar{x}}) \\
& <v_{1}(\bar{x})-\left\|\overline{\bar{a}}_{1}-f_{1}\left(\mathbf{v}^{\mathbf{a}}\right)\right\|^{2} \\
& =v_{1}^{\overline{\bar{a}}_{1}} f\left(\mathbf{v}^{\mathbf{a}}\right),
\end{aligned}
$$

and individual 1 can therefore manipulate $f$ at $\mathbf{v}^{\overline{\bar{a}}}$ via $v_{1}^{\bar{a}_{1}}$, contrary to the nonmanipulability of $f$, thereby completing the lemma's proof.

Now that we know there is some individual $i$ who is an $X$-dictator for $f$ on $\mathscr{U}^{*}$, we can ultimately show that this same individual $i$ is in fact a dictator on all of $\mathscr{U}$. Our method will be to first show, in Lemma 7, that if individual $i$ is an $X$-dictator on $\mathscr{U}^{*}$, then he will still be an $X$-dictator if we expand the domain of profiles from $\mathscr{U}^{*}$ to $U_{i}^{Q} \times \mathscr{U}_{\sim i}$ (recall that $U_{i}^{Q}$ is the set of all functions that satisfy Assumption $1 \mathrm{~b}$ and are strictly concave and quadratic on $X \times Y_{i}$ ). Then we will show, in Lemma 8 , that the same individual is in fact a dictator (not merely an $X$-dictator) on a domain $\tilde{U}_{i} \times \mathscr{U}_{\sim i}$, where $\tilde{U}_{i}$ is a subset of $U_{i}^{Q}$. Then it will be easy to complete the theorem's proof by showing that individual $i$ remains a dictator if the domain is expanded to $\mathscr{U}$.

Lemma 7. Some individual $i$ is an $X$-dictator for $f$ on $U_{i}^{Q} \times \mathscr{U}_{\sim i}$.

Proof. Without loss of generality, let individual 1 be an $X$-dictator for $f$ on $\mathscr{U}^{*}$ (Lemma 6). The proof will proceed in two steps: In Step 1 we will establish that individual 1 is an $X$-dictator on $U_{1}^{*} \times \mathscr{U}_{\sim 1}$, and in Step 2 we will use Step 1 to establish that he is an $X$-dictator on $U_{1}^{Q} \times \mathscr{U}_{\sim 1}$.

Step 1. Let $\mathbf{u} \in U_{1}^{*} \times \mathscr{U}_{\sim 1}$, let $(\bar{x}, \bar{y})=f(\mathbf{u})$, and (since $\left.u_{1} \in U_{1}^{*}\right)$ let $u_{1}=v_{1}^{a_{1}}$. Let $M$ denote the set of those $x \in X$ that maximize $v_{1}$ on the set $f_{x}(\mathscr{U})$; we must show that $\bar{x} \in M$. Suppose to the contrary that $\bar{x} \notin M$, and we will obtain a contradiction.

Let a be a member of $Y$ whose first component is the $a_{1}$ already specified in $v_{1}^{a 1}$. We will recursively define, for $k=1,2, \ldots, n$, profiles $\mathbf{u}^{k}$ which have the form

$$
\mathbf{u}^{k}=\left(v_{1}^{\alpha_{1}}, v_{2}^{\alpha_{2}}, \ldots, v_{k}^{\alpha_{k}}, u_{k+1}, \ldots, u_{n}\right),
$$

and we will define $\left(x^{k}, \mathbf{y}^{k}\right)$ by

$$
\left(x^{k}, \mathbf{y}^{k}\right)=f\left(\mathbf{u}^{k}\right) \text {. }
$$


This construction will be carried out in such a way that, for each $k, x^{k} \notin M$. In particular, $x^{n} \notin M-$ i.e., $f_{x}\left(\mathbf{v}^{\mathbf{a}}\right) \notin M$. But individual 1 is an $X$-dictator on $\mathscr{U}^{*}$; therefore we must in fact have $f_{x}\left(\mathbf{v}^{\mathrm{a}}\right) \in M$, and this contradiction establishes that $\bar{x} \in M$ after all. All that is left, then, is to carry out the recursion - to define, at each $k$, the function $v_{k}$, and to show that $x^{k} \notin M$.

At the first stage of the recursion, i.e., for $k=1,(1)$ and (2) yield $\mathbf{u}^{1}=\mathbf{u}$ and $\left(x^{1}, \mathbf{y}^{1}\right)=(\bar{x}, \bar{y})$. We therefore have $x^{1} \notin M$, as we desired. For $k=2,3, \ldots, n$, let $v_{k}$ be any member of $V$ that satisfies

$$
v_{k}\left(x^{k-1}\right)-v_{k}(x)>\left\|y_{k}^{k-1}-a_{k}\right\|^{2} \text { for every } x \in M \text {. }
$$

The existence of a $v_{k}$ that satisfies (3) is assured by our having established at the preceding stage that $x^{k-1} \notin M$ and because $M$ is closed relative to $f_{x}(\mathscr{U})$. To establish at the current stage that $x^{k} \notin M$, suppose instead that $x^{k} \in M$. Then we have

$$
\begin{aligned}
u_{k}^{k} f\left(\mathbf{u}^{k}\right) & =v_{k}^{a_{k}}\left(x^{k}, \mathbf{y}^{k}\right) \\
& =v_{k}\left(x^{k}\right)-\left\|y_{k}^{k}-a_{k}\right\|^{2}, \quad \text { by definition of } v_{k}^{a_{k}} \\
& \leqq \boldsymbol{v}_{k}\left(x^{k}\right) \\
& <v_{k}\left(x^{k-1}\right)-\left\|y_{k}^{k-1}-a_{k}\right\|^{2}, \quad \text { according to }(3) \\
& =\boldsymbol{v}_{k}^{a_{k}}\left(x^{k-1}, \mathbf{y}^{k-1}\right) \\
& =u_{k}^{k} f\left(\mathbf{u}^{k-1}\right),
\end{aligned}
$$

and individual $k$ would therefore be able to manipulate $f$ at $\mathbf{u}^{k}$ via $u_{k}^{k-1}=u_{k}$, contradicting the nonmanipulability of $f$. Hence, $x^{k} \notin M$, and Step 1 is complete.

Step 2. Let $\mathbf{u} \in U_{1}^{Q} \times \mathscr{U}_{1 \sim}$ and let $(\bar{x}, \overline{\mathbf{y}})$ denote $f(\mathbf{u})$. We must show that $u_{1}(x, \overline{\mathbf{y}}) \leqq u_{1}(\bar{x}, \overline{\mathbf{y}})$ for all $x \in f_{x}\left(U_{1}^{Q} \times \mathscr{U}_{1 \sim}\right)$, which implies that individual 1 is an $X$-dictator for $f$ on $U_{1}^{Q} \times \mathscr{U}_{\sim 1}$. Suppose, to the contrary, that $u_{1}(\hat{x}, \overline{\mathbf{y}})$ $>u_{1}(\bar{x}, \bar{y})$ for some $\hat{x} \in f_{x}\left(U_{1}^{Q} \times \mathscr{U}_{\sim 1}\right)$, and note that Lemma 5 yields $f_{x}\left(U_{1}^{Q} \times \mathscr{U}_{\sim 1}\right)=f_{x}(\mathscr{U})$, because $U_{i}^{*} \subseteq U_{i}^{Q} \subseteq U_{i}$. Then there is a $\delta>0$ for which the following is true of every $y \in Y$ :

$$
\left\|y_{1}-\bar{y}_{1}\right\|^{2}<\delta \Rightarrow u_{1}(\hat{x}, \mathbf{y})>u_{1}(\bar{x}, \overline{\mathbf{y}})
$$

Let $v_{1}$ be a member of $V$ which satisfies both of the following:

$$
\begin{aligned}
& \hat{x} \text { maximizes } v_{1} \text { uniquely on } f_{x}(\mathscr{U}), \\
& v_{1}(\hat{x})-v_{1}(\bar{x})<\delta,
\end{aligned}
$$

and denote $f\left(v_{1}^{\vec{y}_{1}}, \mathbf{u}_{\sim 1}\right)$ by $(\tilde{x}, \tilde{\mathbf{y}})$. Then Step 1 guarantees that $\tilde{x}=\hat{x}$. Furthermore, $\left\|\tilde{y}_{1}-\bar{y}_{1}\right\|^{2}<\delta$, because if $\left\|\tilde{y}_{1}-\bar{y}_{1}\right\|^{2} \geqq \delta$ then we would have 


$$
\begin{aligned}
v_{1}^{\overline{y_{1}}} f\left(v_{1}^{\overline{y_{1}}}, \mathbf{u}_{\sim 1}\right) & =v_{1}(\hat{x})-\left\|\tilde{y}_{1}-\bar{y}_{1}\right\|^{2} \\
& \leqq v_{1}(\hat{x})-\delta \\
& <v_{1}(\bar{x}) \\
& =v_{1}(\bar{x})-\left\|\bar{y}_{1}-\bar{y}_{1}\right\|^{2} \\
& =v_{1}^{\bar{y} 1} f(\mathbf{u}),
\end{aligned}
$$

and individual 1 would therefore be able to manipulate $f$ at $\left(\boldsymbol{v}_{1}^{\overline{\bar{y}_{1}}}, \mathbf{u}_{\sim 1}\right)$ via $u_{1}$. Thus, $\left\|\tilde{y}_{1}-\bar{y}_{1}\right\|^{2}<\delta$, and (4) therefore guarantees that $u_{1}(\hat{x}, \tilde{\mathbf{y}})>u_{1}(\bar{x}, \overline{\mathbf{y}}) ; a$ fortiori, $u_{1}(\tilde{x}, \tilde{\mathbf{y}})>u_{1}(\bar{x}, \overline{\mathbf{y}})$ - i.e., $u_{1} f\left(v_{1}^{\bar{y} 1}, \mathbf{u}_{\sim 1}\right)>u_{1} f(\mathbf{u})$, and individual 1 can therefore manipulate $f$ at $\mathbf{u}$ via $v_{1}^{\bar{y}_{1}}$, contrary to the assumption that $f$ is nonmanipulable. This contradiction establishes that, after all, $u_{1}(\hat{x}, \overline{\mathbf{y}}) \leqq u_{1}(\bar{x}, \overline{\mathbf{y}})$, thereby completing the lemma's proof.

In order to show that an individual $i$ who is an $X$-dictator is actually a dictator, it will be necessary to focus attention on utility functions that have a unique maximizer in the components of interest to the dictator and are not separable in those components. Thus, for each $i \in N$, we let $\tilde{U}_{i}$ denote the set of all utility functions $u_{i}$ in $U_{i}^{Q}$ for which $\dot{u}_{1}$ has a unique maximizer $\left(\hat{x}, \hat{\mathrm{y}}_{1}\right)$ and for which, whenever $y_{1} \neq \hat{y}_{1}, \dot{u}_{1}\left(\cdot, y_{1}\right)$ is not maximized at $\hat{x}$.

Lemma 8. Some individual $i$ is a dictator for $f$ on $\tilde{U}_{i} \times \mathscr{U}_{\sim i}$, and $f\left(\widetilde{U}_{i} \times \mathscr{U}_{\sim i}\right)$ $=f(\mathscr{U})$.

Proof. Without loss of generality, assume that individual 1 is an $X$-dictator for $f$ on $U_{1}^{Q} \times \mathscr{U}_{\sim 1}$ (Lemma 7). We will show that individual 1 is a dictator for $f$ on $\tilde{U}_{1} \times \mathscr{U}_{\sim 1}$, and then that $f\left(\tilde{U}_{1} \times \mathscr{U}_{\sim 1}\right)=f(\mathscr{U})$. Let $u \in \tilde{U}_{1} \times \mathscr{U}_{\sim 1}$; let $(\bar{x}, \bar{y})=f(\mathbf{u})$; and let $(\xi, \eta)$ be a member of $f(\mathscr{U})$ for which $\left(\xi, \eta_{1}\right)$ is the unique maximizer of $\dot{u}_{1}$. Note that we cannot say at this point that $(\xi, \eta) \in f\left(\tilde{U}_{1} \times \mathscr{U}_{\sim 1}\right)$; it is in the second part of the proof that we will establish that $f(\mathscr{U}) \subseteq f\left(\widetilde{U}_{1} \times \mathscr{U}_{\sim 1}\right)$. For now, we will show that $\left(\bar{x}, \overline{\mathrm{y}}_{1}\right)=\left(\xi, \eta_{1}\right)$. It will suffice to show that $\bar{y}_{1}=\eta_{1}$ : Since 1 is an $X$-dictator on $U_{1}^{Q} \times \mathscr{U}_{1 \sim}$ and $\xi$ is the unique maximizer of $\dot{u}_{1}\left(\cdot, \eta_{1}\right)$, we must have $f_{x}(\mathbf{u})=\xi-$ i.e., $\bar{x}_{1}=\xi_{1}-$ whenever $\bar{y}_{1}=\eta_{1}$.

Suppose, then, that $\bar{y}_{1} \neq \eta_{1}$. Then $u_{1}\left(\cdot, \tilde{y}_{1}\right)$ is not maximized at $\xi$, and since individual 1 is an $X$-dictator on $U_{1}^{Q} \times \mathscr{U}_{\sim 1}$, we have $\bar{x}_{1} \neq \xi$. As in Step 1 of the proof of Lemma 7, we recursively define, for $k=2, \ldots, n$, profiles $\mathbf{u}^{k}$ of the form $\mathbf{u}^{k}=\left(u_{1}, v_{2}^{\eta 2}, \ldots, v_{k}^{\eta k}, u_{k+1}, \ldots, u_{n}\right)$ and $\mathbf{u}^{1}=\mathbf{u}$, and we write $\left(x^{k}, \mathbf{y}^{k}\right)=f\left(\mathbf{u}^{k}\right)$, and we carry out the recursive definition in such a way that at each step $k, x^{k} \neq \xi$ : For $k=2, \ldots, n$ let $v_{k}$ be a member of $V$ that satisfies

$$
v_{k}\left(x^{k-1}\right)-v_{k}(\xi)>\left\|y_{k}^{k-1}-\eta_{k}\right\|^{2} \text {. }
$$

As in Step 1 of the proof of Lemma 7, it follows from the nonmanipulability of $f$ that $x^{k} \neq \xi$, and the existence of a $v_{k}$ that satisfies (1) is guaranteed by having shown, at stage $k-1$ of the recursion, that $x^{k-1} \neq \xi$. After the last stage of the recursion, we have $f_{x}\left(u_{1}, \mathbf{v}_{\sim 1}^{\eta}\right)=x^{n} \neq \xi$. Now let $v_{1}$ be a member of $V$ which is maximized over $f_{x}(\mathscr{U})$ at $\tilde{\xi}$, and consider the profile $\mathbf{v}^{\eta}=\left(v_{1}^{\eta_{1}}, \ldots, v_{n}^{\eta_{n}}\right) \in \mathscr{U}^{*}$. Since individual 1 is an $X$-dictator for $f$ on $\mathscr{U}^{*}$, we have $f_{x}\left(\mathbf{v}^{\eta}\right)=\xi$, and Lemma 1 yields $f_{y}\left(\mathbf{v}^{\eta}\right)=\eta$. Therefore $f$ is manipulable by 1 at $\left(u_{1}, \mathbf{v}_{\sim 1}^{\eta} \sim^{1}\right)$ via $v_{1}^{\eta 1}$, contrary 
to the assumption that $f$ is nonmanipulable. This contradiction establishes that $y_{1}=\eta_{1}$, completing the proof that individual 1 is a dictator for $f$ on $\widetilde{U}_{1} \times \mathscr{U}_{\sim 1}$.

We can now show that $f\left(\tilde{U}_{1} \times \mathscr{U}_{\sim 1}\right)=f(\mathscr{U})$. Clearly, $f\left(\tilde{U}_{1} \times \mathscr{U}_{\sim 1}\right) \subseteq f(\tilde{\mathscr{U}})$. To show that $f(\mathscr{U}) \subseteq f\left(\widetilde{U}_{1} \times \mathscr{U}_{\sim 1}\right)$, let $(x, \mathbf{y}) \in f(\mathscr{U})$, and we will show that $(x, \mathbf{y}) \in f\left(\tilde{U}_{1} \times \mathscr{U}_{\sim 1}\right)$. Let $\mathbf{u}$ be a profile in $\tilde{U}_{1} \times \mathscr{U}_{\sim 1}$ for which $\left(x, y_{1}\right)$ is the unique maximizer of $\dot{u}_{1}$ and, for each $i \in\{2, \ldots, n\}, \tilde{y}_{i}$ is the unique maximizer of $\dot{u}_{i}(x, \cdot)$ on $Y_{i}$. Then the first part of the lemma's proof yields $f_{x}(\mathbf{u})=x$, and Lemma 1 yields $f_{y}(\mathbf{u})=\mathbf{y}$ - i.e., $f(\mathbf{u})=(x, \mathbf{y})$ and therefore $(x, \mathbf{y}) \in f\left(\tilde{U}_{1} \times \mathscr{U}_{\sim 1}\right)$.

\section{Proof that $f$ is dictatorial}

Without loss of generality, assume that individual 1 is a dictator for $f$ on $\widetilde{U}_{1} \times \mathscr{U}_{\sim 1}$ (Lemma 8), and suppose that he is not a dictator on $\mathscr{U}$ : let $\mathbf{u} \in \tilde{\mathscr{U}}^{1}$ and $(\hat{x}, \hat{y}) \in f(\mathscr{U})$ be such that $u_{1}(\hat{x}, \hat{y})>u_{1} f(\mathbf{u})$. Let $u_{1}^{\prime}$ be a member of $\widetilde{U}_{1}$ for which $\left(\hat{x}, \hat{y}_{1}\right)$ is the unique maximizer of $\dot{u}_{1}^{\prime}$; then, since individual 1 is a dictator for $f$ on $\tilde{U}_{1} \times \mathscr{U}_{\sim 1}$, and since $(\hat{x}, \hat{y}) \in f(\mathscr{U})=f\left(\tilde{U}_{1} \times \mathscr{U}_{\sim 1}\right)$ it follows that $\left(f_{x}\left(u_{1}^{\prime}, \mathbf{u}_{\sim 1}\right), f_{1}\left(u_{1}^{\prime}, \mathbf{u}_{\sim 1}\right)\right)=\left(\hat{x}, \hat{y}_{1}\right)$, and thus that $u_{1} f\left(u_{1}^{\prime}, \mathbf{u}_{\sim 1}\right)$ $=u_{1}(\hat{x}, \hat{y})>u_{1} f(\mathbf{u})-$ i.e., $f$ is manipulable by individual 1 at $\mathbf{u}$ via $u_{1}^{\prime}$, contrary to the assumption that $f$ is nonmanupulable. Therefore, individual 1 is a dictator for $f$ after all, and the theorem's proof is complete.

The following two examples indicate the importance of certain features of Assumptions 1 and 2. In the first example Assumption 2 is violated - only separable utility functions are admissible - and a nonmanipulable, nondictatorial voting scheme is constructed.

Example 4. Let $n=2$; let $Z=X \times Y$, where $X$ is the unit square $[0,1]^{2}$ and $Y=\left\{y \in \mathbb{R}^{2} \mid y_{1}+y_{2}=1\right\}$; and let each $U_{i}$ contain only separable utility functions, i.e., ones of the form $u_{i}(x, y)=v_{i}(x)+w_{i}\left(y_{i}\right)$. Define the voting scheme $f: \mathscr{U} \rightarrow Z$ as follows: $f_{x}(\mathbf{u})$ maximizes $v_{1} ; f_{1}(\mathbf{u})$ maximizes $w_{2}$; and $f_{2}(\mathbf{u})=1-f_{1}(\mathbf{u})$. This mechanism, while clearly neither manipulable nor dictatorial, is nevertheless rather unsatisfactory: Individual 1 always chooses the value of $x$-i.e., he is an $X$ dictator - and individual 2 always chooses the remaining two ("private") components. In Lemma 8, where an $X$-dictator was shown to be a dictator, a subset $\tilde{U}_{1}$ of $U_{1}$ was constructed which contained certain nonseparable utility functions; this was the only place in the theorem's proof in which nonseparable functions appeared, and it is precisely the absence of the kind of functions in the set $\widetilde{U}_{1}$ that prevents either of the individuals in this example from manipulating the mechanism $f$.

In the next example, as in the theorem, each participant is unaffected by some dimension of the outcome (and these dimensions are linearly independent of one another), but here there are no common dimensions that affect everyone - there are no "purely public' components of the social decision - and we are able to construct a voting scheme that is neither manipulable nor dictatorial. (The voting scheme is nevertheless quite unsatisfactory.)

Example 5. Let $n=3$; let $Z=[0,1]^{3}$, the unit cube in $\mathbb{R}^{3}$, and let each $U_{i}$ consist of all the utility functions $u_{i}$ of the form $u_{i}(\mathbf{x})=\dot{u}_{i}\left(x_{i}, x_{i+1}\right)$, where $i+1=1$ if $i=3$. Define the voting scheme $f: \mathscr{U} \rightarrow Z$ as follows, where $\left(\hat{x}_{i}^{i}, \hat{x}_{i+1}^{i}\right)$ denotes, for each $i$, the (unique) maximizer of $\dot{u}_{i}: f_{2}(\mathbf{u})=\max \left\{\hat{x}_{2}^{\mathrm{l}}, \hat{x}_{2}^{2}\right\} ; f_{1}(\mathbf{u})$ maximizes $\dot{u}_{1}\left(x_{1}, f_{2}(\mathbf{u})\right)$; and $f_{3}(\mathbf{u})$ maximizes $\dot{u}_{2}\left(f_{2}(\mathbf{u}), x_{3}\right)$. The outcome is entirely deter- 
mined by just the utility functions $u_{1}$ and $u_{2}$ : The dimension that affects them both, $x_{2}$, is chosen by a "median voter" rule ( $\min$ would work as well as $\max$ ), and each of the remaining two dimensions is chosen by the individual who cares about it. Individual 3 has no influence on the outcome. The voting scheme $f$ is clearly nonmanipulable; it is also nondictatorial, although from individual 3's point of view the other two participants are "jointly dictating" to him. It is easy to see that similar examples exist for any number of participants, examples in which, for instance, two individuals always "jointly dictate" to everyone else. This example suggests that if our impossibility theorem and its predecessors are, in some form, valid in more general settings, they must include a generalized definition of dictatorship.

We conclude with several additional remarks.

Remark 1. The theorem we have presented contains Zhou's theorem as a special case - the case in which the set $Y$ is a singleton. Note, however, that Zhou's theorem plays an important role in the proof we have given.

Remark 2. The classical allocation problem (viz., the "pure exchange", or "multiperson Edgeworth box" problem) might seem to be the most obvious application of the theorem: One generally assumes in the allocation problem that each of the participants cares only about certain components of the outcomes. But in fact the theorem does not apply to this problem as it is usually formulated. Assumption 2 of the theorem requires that nonmonotone utility functions be admissible, while in the allocation problem all utility functions are usually assumed to be monotone in each component. Example 3 is also worth noting in this regard.

Remark 3. It is not clear whether the CU Property is essential. We have not been able to construct a nonmanipuable, nondictatorial mechanism that does not have the CU Property.

Remark 4. In addition to the generalization suggested by Remark 2 (i.e., the generalization to sets of only monotone utility functions), it is also an open question whether the theorem can be generalized to cases in which the set $Z$ is not a Cartesian product of $X$ and $Y$ - i.e., in which the social decision does not have a "purely public" part which is independent of the decision's other components. Such a generalization would allow for the treatment of additional kinds of externalities, such as, for example, "costly" public decisions - ones in which the choice of $x$ determines which $y$-decisions will actually be available.

\section{References}

Barberá S, Peleg B (1990) Strategy-proof voting schemes with continuous preferences. Soc Choice Welfare 7:31-38

Gibbard A (1973) Manipulation of voting schemes: a general result. Econometrica 41: $587-602$

Satterthwaite MA (1975) Strategy-proofness and Arrow's conditions : existence and correspondence theorems for voting procedures and social welfare functions. J Econ Theory 35 : $1-18$

Zhou L (1991) Impossibility of strategy-proof mechanisms for economic environments. Rev Econ Stud 58: 107-119 